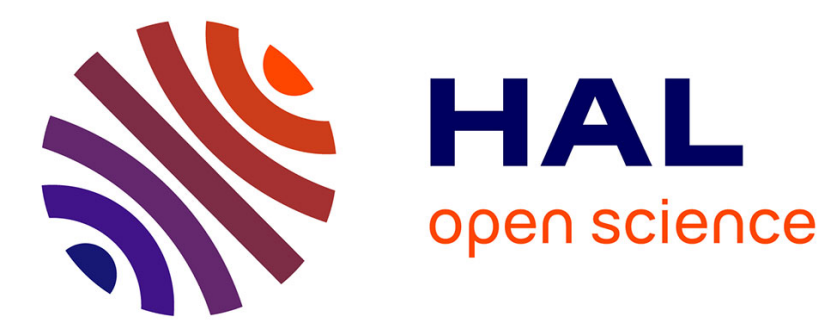

\title{
Structural source identification using a generalized Tikhonov regularization
}

\author{
Mathieu Aucejo
}

\section{To cite this version:}

Mathieu Aucejo. Structural source identification using a generalized Tikhonov regularization. Journal of Sound and Vibration, 2014, 333 (22), pp.5693-5707. 10.1016/j.jsv.2014.06.027 . hal-02068189

\section{HAL Id: hal-02068189 \\ https://hal.science/hal-02068189}

Submitted on 14 Mar 2019

HAL is a multi-disciplinary open access archive for the deposit and dissemination of scientific research documents, whether they are published or not. The documents may come from teaching and research institutions in France or abroad, or from public or private research centers.
L'archive ouverte pluridisciplinaire HAL, est destinée au dépôt et à la diffusion de documents scientifiques de niveau recherche, publiés ou non, émanant des établissements d'enseignement et de recherche français ou étrangers, des laboratoires publics ou privés. 


\title{
Structural source identification using a generalized Tikhonov regularization
}

\author{
M. Aucejo ${ }^{\mathrm{a}}$ \\ ${ }^{a}$ Structural Mechanics and Coupled Systems Laboratory, Conservatoire National des Arts \\ et Métiers, 2 Rue Conté, 75003 Paris, France
}

\begin{abstract}
This paper addresses the problem of identifying mechanical exciting forces from vibration measurements. The proposed approach is based on a generalized Tikhonov regularization that allows taking into account prior information on the measurement noise as well as on the main characteristics of sources to identify like its sparsity or regularity. To solve such a regularization problem efficiently, a Generalized Iteratively Reweighted Least-Squares (GIRLS) algorithm is introduced. Proposed numerical and experimental validations reveal the crucial role of prior information in the quality of the source identification and the performance of the GIRLS algorithm.

Keywords: Source identification, Generalized Tikhonov regularization, Bayesian inference.
\end{abstract}

\section{Introduction}

The control of the vibro-acoustic behavior of structures remains a challenging task in many industrial applications. A possible solution is to control vibration at source. In this situation, the knowledge of excitation sources is

\footnotetext{
*Corresponding author. E-mail address : mathieu.aucejo@cnam.fr
} 
required. However, such information is sometimes difficult or even impossible to measure. A possible alternative to bypass this drawback is to identify excitation sources from vibration measurements. The resolution of this inverse problem has been extensively studied over the past three decades. The most widespread approaches are based on the transfer functions matrix of the structure under test $[1,2,3,4,5]$, that can be numerically computed $[2,6]$ or measured $[7,8,9]$. There exists, however, alternative approaches based on the numerical calculation of a local operator corresponding to the dynamic stiffness of a part of the structure $[10,11,12,13,14]$.

Unfortunately, all the proposed methods are very sensitive to measurement errors causing the identification to fail. That is why, there have been numerous studies focusing on the development of regularization methods to stabilize this inverse problem. Except methods for which very specific procedures have been proposed [10,15], the most popular regularization methods remain the Truncated Singular Value Decomposition (TSVD) [16, 17, 18, 19, 20] and the Tikhonov regularization $[6,13,14,20,21,22]$.

In the present article, we are interested in solving the identification problem using the following generalized Tikhonov regularization [23]:

$$
\left.\widehat{\mathbf{F}}_{\mathbf{c}}=\underset{\mathbf{F}_{\mathbf{c}}}{\operatorname{argmin}} \frac{1}{p}\left\|\mathbf{H F}_{\mathbf{c}}-\mathbf{X}_{\mathbf{m}}\right\|_{p}^{p}+\frac{\lambda}{q}\left\|\mathbf{L F}_{\mathbf{c}}\right\|_{q}^{q}, \quad \forall(p, q) \in\right] 0, \infty\left[^{2},\right.
$$

where $\mathbf{X}_{\mathbf{m}}$ is the vibration field (displacement, velocity or acceleration) measured over the structure, $\mathbf{F}_{\mathbf{c}}$ is the force vector to identify, $\mathbf{H}$ is the corresponding transfer functions matrix, $\|\bullet\|_{p}$ is either the $\ell_{p}$ norm if $\mathrm{p} \geq 1$ or the $\ell_{p}$ quasi-norm if $\mathrm{p}<1$ and $\widehat{\mathbf{F}}_{\mathbf{c}}$ is the particular value of $\mathbf{F}_{\mathbf{c}}$ for which the functional reaches its minimum. Such a problem is said to be convex when $\mathrm{p} \geq 1$ 
and $q \geq 1$ and non-convex otherwise. In the next of the paper, the proposed generalized Tikhonov regularization is also referred to as $\ell_{p}-\ell_{q}$ regularization.

In Eq.(1), the functional to minimize is the sum of two terms. The first one is the data fidelity term $\frac{1}{p}\left\|\mathbf{H F}_{\mathbf{c}}-\mathbf{X}_{\mathbf{m}}\right\|_{p}^{p}$, which is related to measurement noise and allows controlling the a priori on the nature of the noise [24, 25]. The second one is the regularization term $\frac{1}{q}\left\|\mathbf{L F}_{\mathbf{c}}\right\|_{q}^{q}$, that introduces an a priori on the solution, like its regularity thanks to the differentiation matrix L [26], or its sparsity, by using an $\ell_{q}$ norm such as $q<2$ [27]. Finally, these terms are related by the trade-off parameter, $\lambda \in \mathbb{R}^{+}$, that represents the balance between data fidelity and regularization terms. In this formalism, the standard Tikhonov regularization corresponds to $\mathrm{p}=\mathrm{q}=2$. As shown in $[13,14]$, this regularization leads to a systematic smoothing of regularized solutions, which is not a desirable effect when one wants to identify localized sources, for instance.

To have a better understanding of this result, it is worth turning to deconvolution techniques developed in image and signal processing, in which the influence of data fidelity and regularization terms has been deeply studied. It has been shown in [25] that having a data fidelity term reflecting the noise characteristics of the signal provides better reconstruction. In particular, it has been pointed out that using the $\ell_{2}$ norm for the data fidelity term was most appropriate to remove additive gaussian white noise [24, 28], whereas using $\ell_{1}$ norm was suitable for removing impulsive noise [28, 29]. Concerning the regularization term, it has been proven that sparsity-promoting terms allow 
preserving discontinuities in deconvolved signal or image [30, 31, 32, 33, 34]. The most widely used are the regularization terms based on the $\ell_{1}$ norm [30, 31]. However, it has been demonstrated recently that non-convex sparse regularization terms, i.e. based on the $\ell_{q}$ quasi-norm (q $\left.\in\right] 0,1[)$, yield even better discontinuities preservation when compared to the convex $\ell_{1}$ type regularization [35, 36, 37]. To solve efficiently convex as well as non-convex sparse regularization problems, only a few algorithms are available. They are generally based on a generalized version of the Iteratively Reweighted Least-Squares approach (IRLS) [38, 39, 40], originally proposed to deal with problems of the form of Eq.(1) for $\lambda=0$ [41, 42, 43].

To the author's knowledge, the aforementioned methods have been seldom applied in the context of structural source identification. One can nevertheless cite the recent work of Chardon and Daudet [44], in which they apply a convex $\ell_{2}-\ell_{1}$ regularization, processed by a group matching pursuit algorithm, to localize sources acting on a thin plate. Finally, it is important to cite works of Guillaume et al. [8, 9] that propose using an IRLS type procedure to identify localized sources, as well as the work of Renzi et al. [13, 45].

This paper proposes demonstrating the ability of the generalized Tihkonov regularization to identify vibration sources from a GIRLS algorithm. Unlike the standard IRLS procedure, the proposed approach allows introducing, besides prior information on measurement noise, an a priori on the spatial distribution of sources.

To clearly distinguish the main features of the proposed regularization pro- 
cedure, the present article is divided into four parts. In section 2, the general methodology, based on a Finite Element (FE) modelling of a part of the structure under test, is presented. In section 3, the GIRLS algorithm is detailed. A particular attention is paid to the crucial choice of the regularization parameters and the definition of a robust stopping criterion. Finally, sections 4 and 5 are devoted to the numerical and experimental validations of the proposed methodology. Obtained results show that using properly a priori knowledges on the measurement noise and the spatial distribution of the solution improves drastically the quality of the identification and the performance of the GIRLS algorithm.

\section{General principles of the identification process}

As explained in the introduction, one seeks to deal with the structural source identification problem using the generalized Tikhonov regularization given by Eq.(1). To properly solve this problem, one needs to know the mechanical behavior of the part of the structure under test. Such information is given by the transfer functions matrix $\mathbf{H}$. Since the measurement of $\mathbf{H}$ can be quite cumbersome, one prefers using a FE model of the structure instead.

Assuming the structure is linear and the damping is of structural type, the FE model of the structure is of the form:

$$
\left[\mathbf{K}(1+j \eta)-\omega^{2} \mathbf{M}\right] \mathbf{X}(\omega)=\mathbf{D}(\omega) \mathbf{X}(\omega)=\mathbf{F}(\omega)
$$

where $\mathbf{M}$ is the mass matrix, $\mathbf{K}$ is the stiffness matrix, $\mathbf{X}(\omega)$ is the vector containing the considered degrees of freedom (dofs), $\mathbf{F}(\omega)$ is the excitation 
vector, $\eta$ is the structural damping factor, $\mathbf{D}(\omega)$ is the dynamic stiffness matrix of the structure and $\omega$ is the angular frequency.

In Eq.(2), the vibration displacement field $\mathbf{X}(\omega)$ contains two types of degrees of freedom (dofs), namely translation and rotation dofs. However, in practical situations, some dofs, such as rotations, are difficult to measure directly. Considering this experimental fact, the FE model given by Eq.(2) has to be transformed into a model containing only the measurable dofs. Several techniques have been used for that purpose, such as static condensation [21], observation matrix approach [14, 46] or exact dynamic condensation $[11,45,47,48]$. Finally, reduction techniques can be used to avoid the need of data in unmeasured areas, such as the Craig-Bampton reduction [13, 45, 49] or the modal reduction [50].

In this paper, Eq.(2) is transformed using an exact dynamic condensation. The basic idea is to express non-measurable dofs with respect to measurable dofs. To this end, the dynamic stiffness matrix is partitioned into measurable dofs $\mathrm{m}$ and non-measurable dofs $\overline{\mathrm{m}}$. After expressing non-measurable dofs in terms of measurable dofs, one obtains:

$$
\mathrm{D}_{\mathrm{c}} \mathrm{X}_{\mathrm{m}}=\mathrm{F}_{\mathrm{c}} \text {, }
$$

where $\mathbf{D}_{\mathbf{c}}=\mathbf{D}_{\mathbf{m m}}-\mathbf{D}_{\mathbf{m} \overline{\mathbf{m}}} \mathbf{D}_{\overline{\mathbf{m}} \overline{\mathbf{m}}}{ }^{-1} \mathbf{D}_{\overline{\mathbf{m}} \mathbf{m}}$ is the condensed dynamic stiffness and $\mathbf{F}_{\mathbf{c}}=\mathbf{F}_{\mathbf{m}}-\mathbf{D}_{\mathbf{m} \overline{\mathbf{m}}} \mathbf{D}_{\overline{\mathbf{m}} \overline{\mathbf{m}}}{ }^{-1} \mathbf{F}_{\overline{\mathbf{m}}}$ is the equivalent excitation vector acting on the measurable dofs of the structure.

Finally, the transfer functions matrix $\mathbf{H}$ of the part of the structure under 
test is proportional to the inverse of the reduced dynamic stiffness matrix $\mathbf{D}_{\mathbf{c}}$, namely:

$$
\mathbf{H}=\mathbf{D}_{\mathbf{c}}^{-1}
$$

Two comments have to be made concerning Eqs.(3) and (4). First of all, the computation of the transfer functions matrix can be computationally expensive due to the inversion at each frequency of the reduced dynamic stiffness matrix $\mathbf{D}_{\mathbf{c}}$. However, as shown in [10, 13, 14], the identification can be performed locally. This means that only a part of the studied structure can be modelled by using free boundary conditions in the corresponding local finite element model. As a consequence, the size of FE model can be limited, which makes the procedure described above applicable on industrial structures. Furthermore, using a FE model with free boundary conditions gives the possibility of identifying the reaction forces acting at boundaries of the studied structure [14].

Moreover, the transfer functions matrix being generally ill-conditioned, the inverse problem is very sensitive to errors in the measured data set $\mathbf{X}_{\mathbf{m}}$. That is why, regularized formulations, such as Eq.(1), have to be used to stabilize the identification with respect to measurement noise that corrupts input data. To go a little further, it could be noticed that the proposed generalized Tikhonov regularization allows including precisely additional information on the nature of the sources acting on a structure. Incidentally, it is expected to obtain better results than that obtained with a classical regularization method. In particular, it can be shown that the proposed regularized formulation can be seen as a Bayesian regularization using Gen- 
eralized Gaussian priors [23]. Indeed, in the Bayesian paradigm, the posterior probability distribution $P\left(\mathbf{F}_{\mathbf{c}} \mid \mathbf{X}_{\mathbf{m}}\right)$, corresponding to the probability of obtaining the force vector $\mathbf{F}_{\mathbf{c}}$ given a measured vibration field $\mathbf{X}_{\mathbf{m}}$ is written according to Bayes' rule [51, 52]:

$$
P\left(\mathbf{F}_{\mathbf{c}} \mid \mathbf{X}_{\mathbf{m}}\right) \propto P\left(\mathbf{X}_{\mathbf{m}} \mid \mathbf{F}_{\mathbf{c}}\right) P\left(\mathbf{F}_{\mathbf{c}}\right)
$$

where the likelihood function $P\left(\mathbf{X}_{\mathbf{m}} \mid \mathbf{F}_{\mathbf{c}}\right)$ corresponds to the probability of measuring the vibration field $\mathbf{X}_{\mathbf{m}}$ given a force vector $\mathbf{F}_{\mathbf{c}}$, while the prior probability distribution $P\left(\mathbf{F}_{\mathbf{c}}\right)$ represents the a priori knowledge on the unknown excitation field $\mathbf{F}_{\mathbf{c}}$ before measuring $\mathbf{X}_{\mathbf{m}}$. From these definitions, it can be inferred that the likelihood function and the prior probability distribution introduce respectively prior information on the measurement noise and the nature of the excitation sources. Here, $P\left(\mathbf{X}_{\mathbf{m}} \mid \mathbf{F}_{\mathbf{c}}\right)$ and $P\left(\mathbf{F}_{\mathbf{c}}\right)$ are supposed to be generalized Gaussian distributions defined such that:

$$
P\left(\mathbf{X}_{\mathbf{m}} \mid \mathbf{F}_{\mathbf{c}}\right) \propto e^{-1 / p\left\|\mathbf{H F}_{\mathbf{c}}-\mathbf{X}_{\mathbf{m}}\right\|_{p}^{p} / \alpha^{p}} \quad \text { and } \quad P\left(\mathbf{F}_{\mathbf{c}}\right) \propto e^{-1 / q\left\|\mathbf{L F}_{\mathbf{c}}\right\|_{q}^{q} / \beta^{q}},
$$

where $\alpha$ and $\beta$ are the scale parameters of the generalized Gaussian distributions.

In the Bayesian philosophy, the most probable force vector $\widehat{\mathbf{F}}_{\mathbf{c}}$ is the maximum a posteriori estimate, that is:

$$
\widehat{\mathbf{F}}_{\mathbf{c}}=\underset{\mathbf{F}_{\mathbf{c}}}{\operatorname{argmax}} P\left(\mathbf{X}_{\mathbf{m}} \mid \mathbf{F}_{\mathbf{c}}\right) P\left(\mathbf{F}_{\mathbf{c}}\right) .
$$

Then, it can be easily shown that the solution $\widehat{\mathbf{F}}_{\mathbf{c}}$ is also the solution of dual minimization problem given by Eq. (1) with $\lambda=\alpha^{p} / \beta^{q}$. The wellknown conclusion of the Bayesian inference [53] is that the more the prior 
information is accurate, the more the posterior probability is high. From this observation, it is expected that accurate results can be obtained if the trade-off parameters $\lambda$ and the tuning parameters $p$ and $q$ are properly chosen, since the regularization process gives the most probable solution given prior information on the measurement noise and the nature of the sources to identify.

At this stage of the identification process, the local transfer functions matrix $\mathbf{H}$ and the vibration field $\mathbf{X}_{\mathbf{m}}$, measured on the corresponding part of the real structure, can be introduced in Eq.(1) to solve the identification problem. To clearly distinguish measurement stages from numerical processing stages, an overview of the whole identification procedure is proposed under a block diagram form in Fig. 1.

The proposed block diagram shows that the general philosophy of the identification process shares some common points with previous works published in the source identification literature, such as the use of a finite element description of the structure $[20,49,50]$ and the condensation of nonmeasurable dofs $[11,13,21]$. Furthermore, it highlights the central role of the regularization step in the whole process, since it allows gathering and postprocessing numerical and experimental input data to provide an estimate of the force vector. Consequently, one needs to use a robust solver to deal with both convex and non-convex minimization problems that can arise when using a generalized Tikhonov regularization. For this purpose, one proposes to use the GIRLS algorithm. 


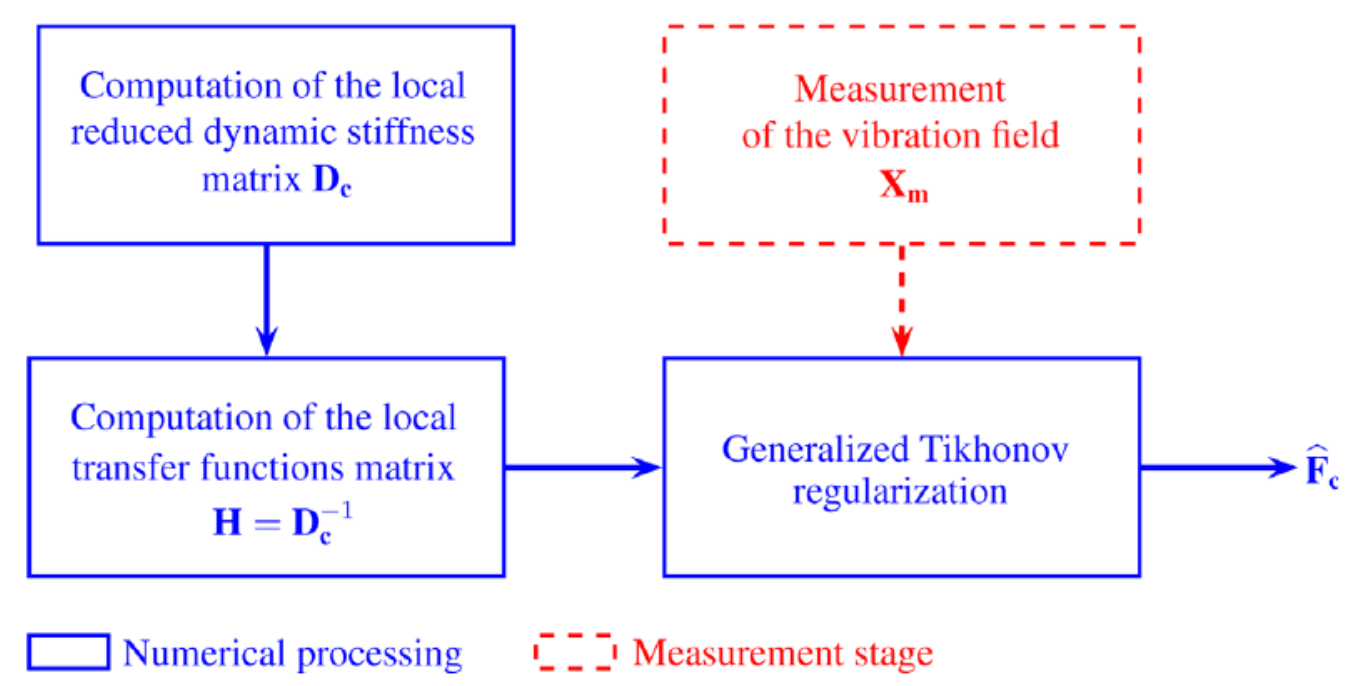

Figure 1: Block diagram of the whole identification procedure

\section{Generalized Iteratively Reweighted Least-Squares algorithm}

The GIRLS approach is a very general iterative procedure that allows solving $\ell_{p}-\ell_{q}$ regularization problems for all $\left.(p, q) \in\right] 0,+\infty[2$.

\subsection{General algorithm}

The core idea of the GIRLS algorithm is to replace the $\ell_{p}-\ell_{q}$ regularization by an equivalent iterative procedure based on a weighted $\ell_{2}-\ell_{2}$ functional. Indeed, it has been shown in $[38,54,40,43]$ that the $\ell_{p}$ norm can be approximated, within an iterative scheme, by a weighted $\ell_{2}$-norm. This means that the functional

$$
J_{p}^{q}\left(\mathbf{F}_{\mathbf{c}}\right)=\frac{1}{p}\left\|\mathbf{H F}_{\mathbf{c}}-\mathbf{X}_{\mathbf{m}}\right\|_{p}^{p}+\frac{\lambda}{q}\left\|\mathbf{L F}_{\mathbf{c}}\right\|_{q}^{q},
$$


is replaced, at each iteration $\mathrm{k}+1$ of an iterative process, by the equivalent quadratic functional

$$
\tilde{J}_{2}^{2}\left(\mathbf{F}_{\mathbf{c}}\right)=\frac{1}{2}\left\|\mathbf{W}_{\mathbf{f}}^{(k)^{1 / 2}}\left(\mathbf{H F}_{\mathbf{c}}-\mathbf{X}_{\mathbf{m}}\right)\right\|_{2}^{2}+\frac{\lambda^{(k+1)}}{2}\left\|\mathbf{W}_{\mathbf{r}}^{(k)^{1 / 2}} \mathbf{L} \mathbf{F}_{\mathbf{c}}\right\|_{2}^{2},
$$

where $\lambda^{(k+1)}$ is the trade-off parameter at iteration $\mathrm{k}+1$, while $\mathbf{W}_{\mathbf{f}}^{(k)}$ and $\mathbf{W}_{\mathbf{r}}^{(k)}$ are weighting positive definite matrices, defined as follows [38]:

$$
\mathbf{W}_{\mathbf{f}}^{(k)}=\operatorname{diag}\left[\frac{2}{p} T_{\epsilon_{f}}^{p}\left(\mathbf{H} \widehat{\mathbf{F}}_{\mathbf{c}}^{(k)}-\mathbf{X}_{\mathbf{m}}\right)\right] \text { and } \mathbf{W}_{\mathbf{r}}^{(k)}=\operatorname{diag}\left[\frac{2}{q} T_{\epsilon_{r}}^{q}\left(\mathbf{L} \widehat{\mathbf{F}}_{\mathbf{c}}^{(k)}\right)\right] \text {, }
$$

where $\widehat{\mathbf{F}}_{\mathbf{c}}^{(k)}$ is the solution at iteration $\mathrm{k}$.

As classically done in IRLS type algorithm [38, 55], the functions $T_{\epsilon_{f}}^{p}\left(x^{(k)}\right)$ and $T_{\epsilon_{r}}^{q}\left(x^{(k)}\right)$ are expressed as

$$
T_{\epsilon_{i}}^{j}\left(x^{(k)}\right)= \begin{cases}\left|x^{(k)}\right|^{j-2} & \text { if }\left|x^{(k)}\right|>\epsilon_{i}^{(k)} \\ \left|\epsilon_{i}^{(k)}\right|^{j-2} & \text { if }\left|x^{(k)}\right| \leq \epsilon_{i}^{(k)}\end{cases}
$$

where $i=f$ or $r$ and $j=p$ or $q$.

In Eq.(11), $\epsilon_{f}^{(k)}$ and $\epsilon_{r}^{(k)}$ are small positive numbers acting as damped parameters. They allows avoiding infinite weights when $\left|x^{(k)}\right| \rightarrow 0$ and $j<2$.

As a result, the solution at iteration $\mathrm{k}+1$ is obtained by solving the equivalent minimization problem:

$$
\widehat{\mathbf{F}}_{\mathbf{c}}^{(k+1)}=\underset{\mathbf{F}_{\mathbf{c}}}{\operatorname{argmin}} \tilde{J}_{2}^{2}\left(\mathbf{F}_{\mathbf{c}}\right)
$$


By expanding the equivalent quadratic functional $\tilde{J}_{2}^{2}\left(\mathbf{F}_{\mathbf{c}}\right)$ and setting its gradient to zero, Eq.(12) has the following explicit form:

$$
\widehat{\mathbf{F}}_{\mathbf{c}}^{(k+1)}=\left(\mathbf{H}^{H} \mathbf{W}_{\mathbf{f}}^{(k)} \mathbf{H}+\lambda^{(k+1)} \mathbf{L}^{H} \mathbf{W}_{\mathbf{r}}^{(k)} \mathbf{L}\right)^{-1} \mathbf{H}^{H} \mathbf{W}_{\mathbf{f}}^{(k)} \mathbf{X}_{\mathbf{m}}
$$

where $\mathbf{H}^{H}$ is the hermitian transpose of $\mathbf{H}$.

The resulting procedure therefore consists in solving Eq.(13) iteratively until some stopping criterion is satisfied. Supposing now the stopping criterion is well chosen. In this situation, if the functional $J_{p}^{q}\left(\mathbf{F}_{\mathbf{c}}\right)$ is convex and possesses a local minimum, then it is a global minimum. However, when dealing with non-convex sparse regularization, the final solution $\widehat{\mathbf{F}}_{\mathbf{c}}$ strongly depends on the initial guess $\widehat{\mathbf{F}}_{\mathbf{c}}^{(0)}$, since the corresponding non-convex functional does not necessarily have an unique minimizer. Consequently, one of the keys for a successful identification is finding a good initial guess. The question that arises here is: What is a good initial guess? Actually, it is a coarse solution of the problem, easy to calculate, but sufficiently close to the final solution to ensure the convergence of the iterative process. Such requirements are fulfilled by the solution of the standard Tikhonov regularization, that is:

$$
\widehat{\mathbf{F}}_{\mathbf{c}}^{(0)}=\underset{\mathbf{F}_{\mathbf{c}}}{\operatorname{argmin}} \frac{1}{2}\left\|\mathbf{H F}_{\mathbf{c}}-\mathbf{X}_{\mathbf{m}}\right\|_{2}^{2}+\frac{\lambda^{(0)}}{2}\left\|\mathbf{L F}_{\mathbf{c}}\right\|_{2}^{2},
$$

or in a more explicit form:

$$
\widehat{\mathbf{F}}_{\mathbf{c}}^{(0)}=\left(\mathbf{H}^{H} \boldsymbol{H}+\lambda^{(0)} \mathbf{L}^{H} \mathbf{L}\right)^{-1} \mathbf{H}^{H} \mathbf{X}_{\mathbf{m}}
$$

All the previous comments lead to the generic GIRLS algorithm given below: 


\section{Generic GIRLS Algorithm}

Inputs: Transfer functions matrix: $\mathbf{H}$, Measured vibration field: $\mathbf{X}_{\mathbf{m}}$ Differentiation matrix: $\mathbf{L}$

Output: Estimated force vector: $\widehat{\mathbf{F}}_{\mathbf{c}}$

Initialization: Compute $\widehat{\mathbf{F}}_{\mathbf{c}}^{(0)}$ from Eq.(15)

\section{Iteration:}

while Stopping criterion is not satisfied

Compute $\mathbf{W}_{\mathbf{f}}^{(k)}$ and $\mathbf{W}_{\mathbf{r}}^{(k)}$ from Eq.(10)

Compute $\widehat{\mathbf{F}}_{\mathbf{c}}^{(k+1)}$ from Eq.(13)

end

$\operatorname{return} \widehat{\mathbf{F}}_{\mathbf{c}} \leftarrow \widehat{\mathbf{F}}_{\mathbf{c}}^{(k)}$ 


\subsection{Choice of the tuning parameters $p$ and $q$}

To choose proper values of $\mathrm{p}$ and $\mathrm{q}$, it is worth reminding some important results established in various contexts such as image deconvolution, signal denoising or convex optimization, already cited in the introduction of the paper. First, it has been proven that using an $\ell_{2}$-norm for the data fidelity term is suitable for removing additive Gaussian white noise [24, 28], while using an $\ell_{1}$-norm is most appropriate to suppress impulsive noise [28, 29]. Then, it has been shown that using an $\ell_{2}$-norm for the regularization term is perfectly adapted to smooth solutions [27], while using a non-convex regularization based on the $\ell_{q}$ quasi-norm $(q \in] 0,1[)$ promotes the sparsity of the solution [56]. In short, selected values have to reflect the main characteristics of the measurement noise and the spatial distribution of sources. Thanks to the results recalled above and a set of numerical experiments, it is possible to give some general rules to properly choose the values of $p$ and $q$. On one hand, in most of the cases, the noise corrupting the data is not known. As a result, a good choice of $p$ is $p=2$, meaning that the noise is assumed to be an additive Gaussian white noise. This is a reasonable assumption, since the noise generally results from a variety of causes. However, when the measurement noise is not additive Gaussian, it is preferable to use $p<2$, with the special case of impulsive noise for which $p=1$. On the other hand, the choice of the value of the tuning parameter $q$ is less obvious. Nevertheless, one can notice that if the sources exciting the structure are smooth, then one can choose $q=2$. On contrary, if the source vector to identify is sparse, it is

recommended to choose $q \leq 1$. Finally, in a situation where actual sources combine both localized and distributed sources, we can choose $q \in] 1,2[$. 


\subsection{Automatic selection of the regularization parameters}

Three iteration-dependent regularization parameters are involved in the GIRLS algorithm, namely $\lambda^{(k)}, \epsilon_{f}^{(k)}$ and $\epsilon_{r}^{(k)}$. Their role in the iterative process is fundamental, since $\lambda^{(k)}$ represents the trade-off between data-fidelity and regularization terms, while $\epsilon_{f}^{(k)}$ and $\epsilon_{r}^{(k)}$ controls the trajectory of each iteration. Choosing manually appropriate values for these parameters is not an easy task. That is why, the development of automatic selection procedures has been extensively studied in the literature.

The automatic selection of the trade-off parameter is at heart of numerous studies dealing with the Tikhonov regularization. Among all the automatic selection methods, one can cite the Morozov's discrepancy principle [57], the Generalized Cross Validation [58], the Unbiased Predictive Risk Estimator [59], the Bayesian estimator [52] and the L-curve method [60]. In the present paper, the trade-off parameter $\lambda^{(k)}$ is obtained using the L-curve principle at each iteration. In few words, the principle is to plot, in a log-log scale, the $l_{2}$ norm of the regularization term versus the $l_{2}$ norm of the data fidelity term. The resulting L-shaped curve illustrates the trade-off between the two terms [see Fig.(2)]. The optimal regularization parameter is derived from the L-curve by calculating its maximum curvature.

Unfortunately, using the L-curve principle to determine the optimal tradeoff parameter $\lambda^{(k)}$ at each iteration is computationally intensive. It can thus be of great interest to use an unique value for this parameter throughout the iterative process. From a series of numerical experiments, we have found that using $\lambda^{(k)}=\lambda^{(0)}$ gives similar results to those obtained from updated 


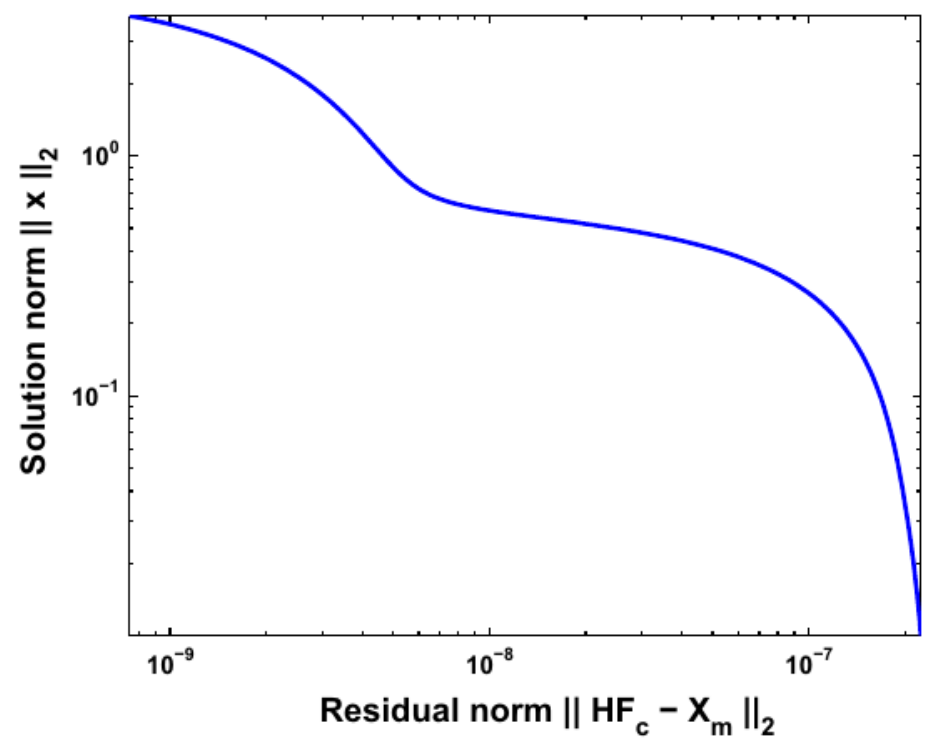

Figure 2: Typical L-curve

$\lambda^{(k)}$ within a comparable number of iterations. Consequently, the trade-off parameter $\lambda^{(k)}$ is chosen once for all during the initialization step.

Concerning the automatic selection of $\epsilon_{f}^{(k)}$ and $\epsilon_{r}^{(k)}$, it has been shown in [54] that their optimal values were functions of the current solution. To adapt automatically the value of $\epsilon_{f}^{(k)}$ (resp. $\epsilon_{r}^{(k)}$ ) at each iteration, it has been proposed to set its value so that a fixed percentage $\mathrm{p}_{\mathrm{f}}$ (resp. $\mathrm{p}_{\mathrm{r}}$ ) of the smallest values of $\left|\mathbf{H} \widehat{\mathbf{F}}_{\mathbf{c}}^{(k)}-\mathbf{X}_{\mathbf{m}}\right|$ (resp. $\left|\mathbf{L} \widehat{\mathbf{F}}_{\mathbf{c}}^{(k)}\right|$ ) will be below $\epsilon_{f}^{(k)}$ (resp. $\epsilon_{r}^{(k)}$ ). This can be easily implemented by calculating the cumulative histogram of $\left|\mathbf{H} \widehat{\mathbf{F}}_{\mathbf{c}}^{(k)}-\mathbf{X}_{\mathbf{m}}\right|$ (resp. $\left|\mathbf{L} \widehat{\mathbf{F}}_{\mathbf{c}}^{(k)}\right|$ ) will be below $\epsilon_{f}^{(k)}\left(\right.$ resp. $\left.\epsilon_{r}^{(k)}\right)$. From our own numerical tests, we have found that setting $\mathrm{p}_{\mathrm{f}}=\mathrm{p}_{\mathrm{r}}=5 \%$ allows obtaining good results. It can be notice that these values are consistent with those proposed in [54], namely $\mathrm{p}_{\mathrm{f}} \leq 5 \%$ and $\mathrm{p}_{\mathrm{r}} \leq 1 \%$. 
As for the automatic selection of the trade-off parameter $\lambda^{(k)}$, accurate solutions can be obtained using fixed values of $\epsilon_{f}^{(k)}$ and $\epsilon_{r}^{(k)}$. From a set of numerical experiment, we have found that using the values computed from the solution of the Tikhonov regularization has no significant impact on both the quality of the results and the time-performance of the algorithm. Accordingly, the regularization parameters $\epsilon_{f}^{(k)}$ and $\epsilon_{r}^{(k)}$ can also be computed once for all during the initialization step.

\subsection{Stopping criterion}

The GIRLS algorithm being iterative, a practical test is needed to determine when to stop the iteration. In this paper, a stopping criterion related to the variation of the functional $\tilde{J}_{2}^{2}\left(\mathbf{F}_{\mathbf{c}}\right)$ between two successive iterations is used.

In an iterative process, it is expected that

$$
\left|\tilde{J}_{2}^{2}\left(\widehat{\mathbf{F}}_{\mathbf{c}}^{(k)}\right)-\tilde{J}_{2}^{2}\left(\widehat{\mathbf{F}}_{\mathbf{c}}^{(k-1)}\right)\right| \approx 0
$$

when the convergence is reached.

From this observation, one defines the normalized variation $\delta$ such that:

$$
\delta=\frac{\left|\tilde{J}_{2}^{2}\left(\widehat{\mathbf{F}}_{\mathbf{c}}^{(k)}\right)-\tilde{J}_{2}^{2}\left(\widehat{\mathbf{F}}_{\mathbf{c}}^{(k-1)}\right)\right|}{\left|\tilde{J}_{2}^{2}\left(\widehat{\mathbf{F}}_{\mathbf{c}}^{(1)}\right)-J_{2}^{2}\left(\widehat{\mathbf{F}}_{\mathbf{c}}^{(0)}\right)\right|},
$$

where $2 J_{2}^{2}\left(\widehat{\mathbf{F}}_{\mathbf{c}}^{(0)}\right)=\left\|\mathbf{H} \widehat{\mathbf{F}}_{\mathbf{c}}^{(0)}-\mathbf{X}_{\mathbf{m}}\right\|_{2}^{2}+\lambda^{(0)}\left\|\mathbf{L} \widehat{\mathbf{F}}_{\mathbf{c}}^{(0)}\right\|_{2}^{2}$. 
If the iterative process is monotonically convergent, the variation between the initialization step and the first iteration is the larger possible one, insofar

as $J_{2}^{2}\left(\widehat{\mathbf{F}}_{\mathbf{c}}^{(0)}\right)$ is the value of the functional for the coarsest solution. Consequently, one has $0 \leq \delta \leq 1$. This indicator allows controlling the behavior of the algorithm, since the iterative process is stopped when $\delta$ is smaller than or equal to a prescribed tolerance defined by the user. Experimentally, we have found that setting the tolerance to $10^{-8}$ leads to accurate regularized solutions, while preserving the time-performance of the GIRLS algorithm.

\subsection{Proposed GIRLS algorithm}

By including the automatic selection procedures of regularization parameters and the stopping criterion previously described in the generic GIRLS algorithm, one obtains the following GIRLS algorithm:

\section{Numerical validation}

In this section, the ability of the GIRLS algorithm to deal with generalized Tikhonov regularization problems is assessed from the identification of a mechanical point force exciting a simply supported plate. The force is supposed acting perpendicularly to the plate. The main characteristics of the test case are given in Table 1.

The FE mesh of the whole plate is made up of 567 linear quadrilateral shell elements. Assuming that only bending motions are measurable, the resulting FE model, obtained from MSC Nastran, is used to compute the corresponding noiseless vibration displacement field $\mathbf{X}_{\mathbf{m}}^{\text {exact }}$. In practical situations, the exact vibration field is corrupted by measurement noise, causing the identification to fail due to the extreme sensitivity of the inverse problem 


\section{Proposed GIRLS Algorithm}

Inputs: Transfer functions matrix: $\mathbf{H}$, Measured vibration field: $\mathbf{X}_{\mathbf{m}}$,

Differentiation matrix: L, Tolerance: tol

Output: Estimated force vector: $\widehat{\mathbf{F}}_{\mathbf{c}}$

Initialization: Compute $\lambda^{(0)}$ from the L-curve principle

Compute $\widehat{\mathbf{F}}_{\mathbf{c}}^{(0)}$ from Eq.(15)

Compute $\epsilon_{f}^{(0)}$ from the cumulative histogram of $\left|\mathbf{H} \widehat{\mathbf{F}}_{\mathbf{c}}^{(0)}-\mathbf{X}_{\mathbf{m}}\right|$

Compute $\epsilon_{r}^{(0)}$ from the cumulative histogram of $\left|\mathbf{L} \widehat{\mathbf{F}}_{\mathbf{c}}^{(0)}\right|$

Initialize $\delta$ to 1

\section{Iteration:}

while $\delta>$ tol

Compute $\mathbf{W}_{\mathbf{f}}^{(k)}$ and $\mathbf{W}_{\mathbf{r}}^{(k)}$ from Eq.(10) using $\epsilon_{f}^{(k)}=\epsilon_{f}^{(0)}$ and $\epsilon_{r}^{(k)}=\epsilon_{r}^{(0)}$

Compute $\widehat{\mathbf{F}}_{\mathbf{c}}^{(k+1)}$ from Eq.(13) using $\lambda^{(k+1)}=\lambda^{(0)}$

Update $\delta$ using Eq.(17)

end

$\operatorname{return} \widehat{\mathbf{F}}_{\mathbf{c}} \leftarrow \widehat{\mathbf{F}}_{\mathbf{c}}^{(k)}$ 
Table 1: Simulation parameters of test case 2

\begin{tabular}{ll}
\hline Parameters & Values \\
\hline Plate length & $L=2 \mathrm{~m}$ \\
Plate width & $b=1.5 \mathrm{~m}$ \\
Plate thickness & $h=0.017 \mathrm{~m}$ \\
Young's modulus & $E=2.1 \times 10^{11} \mathrm{~Pa}$ \\
Density & $\rho=7800 \mathrm{~kg}^{-3}{ }^{-3}$ \\
Structural damping & $\eta=0.01$ \\
Amplitude of the force & $F_{0}=1 \mathrm{~N}$ \\
Location of the force & $\left(x_{0}, y_{0}\right)=(1.04 \mathrm{~m}, 0.93 \mathrm{~m})$ \\
Studied frequency & $f_{0}=350 \mathrm{~Hz}$ \\
\hline
\end{tabular}

to uncertainties. To highlight this phenomenon and assess the efficiency of generalized Tikhonov regularization to solve this problem, the exact data are corrupted by introducing a multiplicative noise $[10,61]$ and an addtive noise [62] to simulate calibration errors and measurement noise respectively:

$$
\widetilde{\mathbf{X}}_{\mathbf{m}}=\boldsymbol{\beta}_{\mathbf{m}} e^{j \phi_{\mathbf{m}}} \mathbf{X}_{\mathbf{m}}^{\text {exact }}+\boldsymbol{\beta}_{\mathbf{a}} e^{j \phi_{\mathbf{a}}},
$$

where $\boldsymbol{\beta}_{\mathbf{m}}=1+p_{n}\left|\mathbf{X}_{\mathbf{m}}^{\text {exact }}\right| \mathbf{Z}_{\mathbf{1}}, \boldsymbol{\phi}_{\mathbf{m}}=\arctan \left(p_{n}\right) \mathbf{Z}_{\mathbf{2}}, \boldsymbol{\beta}_{\mathbf{a}}=p_{n} M^{-1 / 2}\left\|\mathbf{X}_{\mathbf{m}}^{\text {exact }}\right\|_{2} \mathbf{Z}_{\mathbf{3}}$ and $\phi_{\mathbf{a}}=\mathbf{U}$, with $p_{n}$ being the noise percentage, $M$ being the number of measurable dofs, $\left(\mathbf{Z}_{\mathbf{1}}, \mathbf{Z}_{\mathbf{2}}, \mathbf{Z}_{\mathbf{3}}\right)$ being three independent Gaussian random vectors and $\mathbf{U}$ being a random vector uniformly distributed over the interval $[0,2 \pi]$. In what follows, the noise percentage $p_{n}$ is set to $2 \%$.

Finally, a local model of the plate is used to compute the transfer functions matrix $\mathbf{H}$ of measurable dofs. As suggested in [13, 14], this local model corresponds to the FE model with free boundary conditions of a part of the 
structure. In this example, the local model covers the whole extent of the plate, which has the advantage of enabling the identification of external excitations acting on the structure as well as reaction forces at boundaries. In the present case, it should be noted that $\mathbf{H}$ is computed by using the value of the structural damping defined in Table 1.

The reference force vector $\mathbf{F}_{\mathbf{c}}^{\mathrm{ref}}$ is obtained from the condensed dynamic stiffness $\mathbf{D}_{\mathbf{c}}$ of the local model and the exact vibration displacement field $\mathbf{X}_{\mathbf{m}}^{\text {exact }}$ thanks to the following equation:

$$
\mathbf{F}_{\mathbf{c}}^{\mathrm{ref}}=\mathbf{D}_{\mathbf{c}}^{-1} \mathbf{X}_{\mathbf{m}}^{\text {exact }}
$$

Fig. 3 presents the reference force vector to identify at $350 \mathrm{~Hz}$. As expected, a unit point force appears at $\left(\mathrm{x}_{0}, \mathrm{y}_{0}\right)=(1.04 \mathrm{~m}, 0.93 \mathrm{~m}) \mathrm{m}$ as well as smooth reacting forces at the boundaries of the plate.

As presented in Fig. 4, when one replaces exact data $\mathbf{X}_{\mathbf{m}}^{\text {exact }}$ by the corrupted ones $\widetilde{\mathbf{X}}_{\mathbf{m}}$ in Eq. (19), the identification of the force vector fails. This figure emphasizes the extreme sensitivity of the identification problem with respect to measurement noise and allows explaining the need for a regularization process.

The first idea to stabilize the source identification is to use the standard Tikhonov regularization, corresponding to Eq. (1) where $p=q=2$ and $\mathbf{L}$ is the identity matrix I. The corresponding result, presented in Fig. 5(a), shows that the localization of the point force is properly estimated while the amplitude is greatly underestimated. On contrary, reaction forces at boundaries are well identified. This contrasted result can be explained by the use of the $\ell_{2}$-norm for the regularization term that promotes smooth solutions, which is 


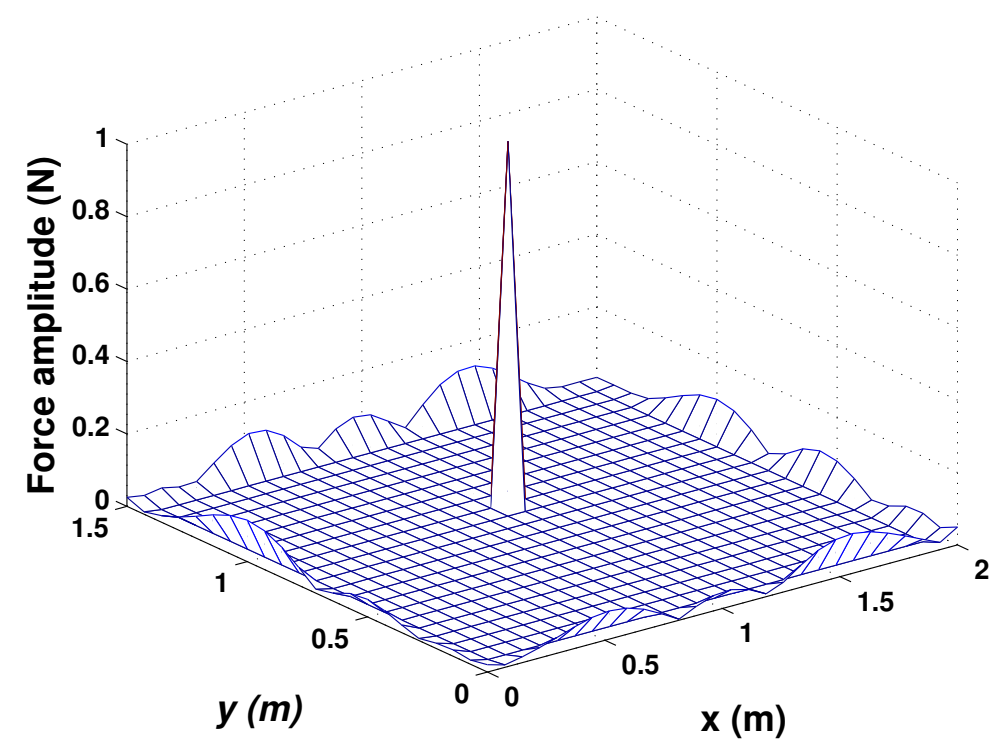

Figure 3: Reference force vector $\mathbf{F}_{\mathbf{c}}^{\text {ref }}$ at $350 \mathrm{~Hz}$

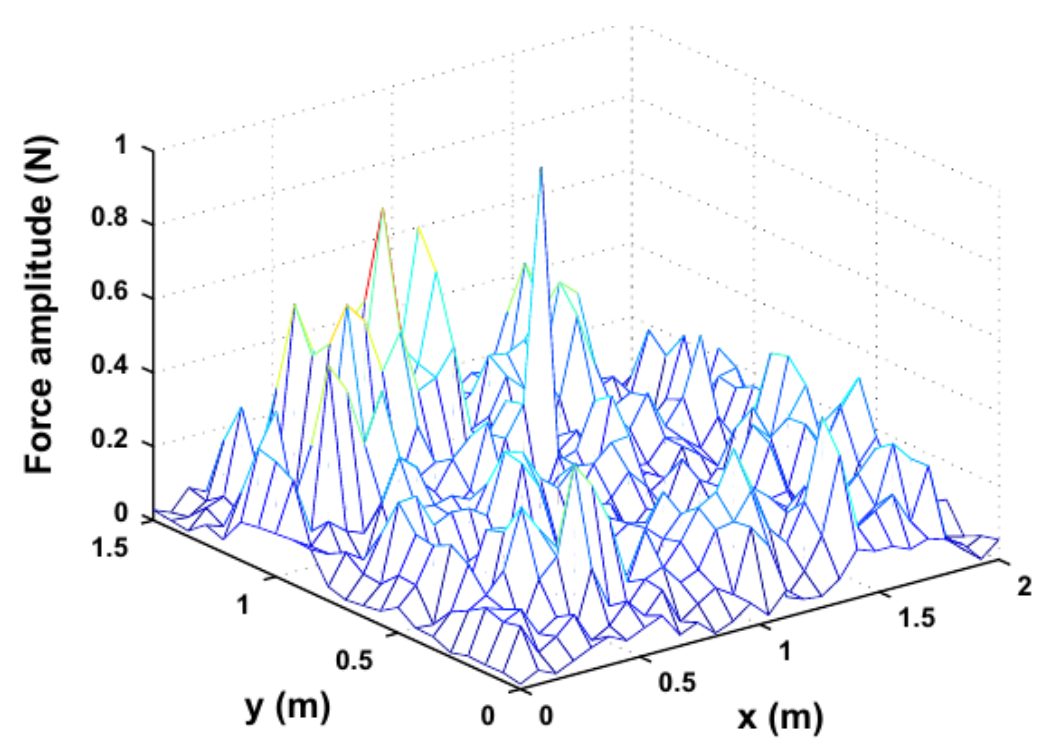

Figure 4: Identification of the force vector $\widehat{\mathbf{F}}_{\mathbf{c}}$ at $350 \mathrm{~Hz}$ from corrupted data and without regularization 
the case for reaction forces but not for the point force. A better identification of the point force can be obtained by using a sparsity-promoting regularization term. This can be achieved from an $\ell_{2}-\ell_{1}$ regularization in which $\mathbf{L}=\mathbf{I}$. Unlike the standard Tikhonov regularization, the $\ell_{2}-\ell_{1}$ regularization allows well identifying the point force, but not the reaction forces at boundaries [see Fig. 5(b)]. In fact, this is the logical outcome of the actual spatial distribution of vibration sources over the plate, that is smooth along the edges, while the solution is supposed to be sparse. Hence, a great number of iterations are necessary to obtain a solution compatible with the prior information. In this example, the $\ell_{2}-\ell_{1}$ regularization regularization has required 80 iterations to reach the tolerance. A faster convergence can be achieved by using properly our a priori on the measurement noise and the target force vector. Indeed, one knows not only that the noise is a combination of multiplicative and additive random noises, but also that the force vector to identify is very sparse, except at boundaries where the force vector is smooth. Regarding the regularization term, a compromise has to be found between the smoothness of the reaction forces at boundaries and the non-smoothness of the point force. Considering the characteristics of the measurement noise, the actual spatial distribution of sources as a prior information and the general rules given in section 3.2, a consistent result is found after 61 iterations using a convex $\ell_{1.9}-\ell_{1.1}$ regularization [see Fig. $5(\mathrm{c})$ ]. These results are consistent with the remark done in section 2 concerning the relation between the proposed formulation and the Bayesian regularization, since the more the prior information on the measurement noise and the spatial distribution of the solution is accurate, the more a reliable regularized solution can be obtained. From a 
computational point of view, the immediate consequence is that the GIRLS algorithm converges within a few iterations.

Finally, it is interesting to notice that the L-curve used to determine the trade-off parameter has the typical aspect of an L-curve as shown in

\section{Experimental validation}

This section aims at assessing the relevance of the proposed approach in operating conditions and confirm the conclusions drawn in the previous numerical validation. As presented in Fig. 6, the structure of interest is a steel parallelepiped box, excited on one of its faces by a shaker equipped with a force sensor. The main features of the proposed experimental validation are listed in table 2.

Table 2: Experiment parameters

\begin{tabular}{ll}
\hline Parameters & Values \\
\hline Length of the parallelepiped & $L_{x}=0.45 \mathrm{~m}$ \\
Width of the parallelepiped & $L_{y}=0.3 \mathrm{~m}$ \\
Height of the parallelepiped & $L_{z}=0.35 \mathrm{~m}$ \\
Wall thickness & $h=0.005 \mathrm{~m}$ \\
Young's modulus & $E=2.1 \times 10^{11} \mathrm{~Pa}$ \\
Density & $\rho=7800 \mathrm{~kg} \cdot \mathrm{m}^{-3}$ \\
Location of the force & $\left(x_{0}, y_{0}, z_{0}\right)=(0 \mathrm{~m}, 0.11 \mathrm{~m}, 0.09 \mathrm{~m})$ \\
Studied frequency & $f_{0}=350 \mathrm{~Hz}$ \\
\hline
\end{tabular}

In this experiment, the source identification has been performed on the excited surface only [see Fig 6]. Measurements of the vibration field were 


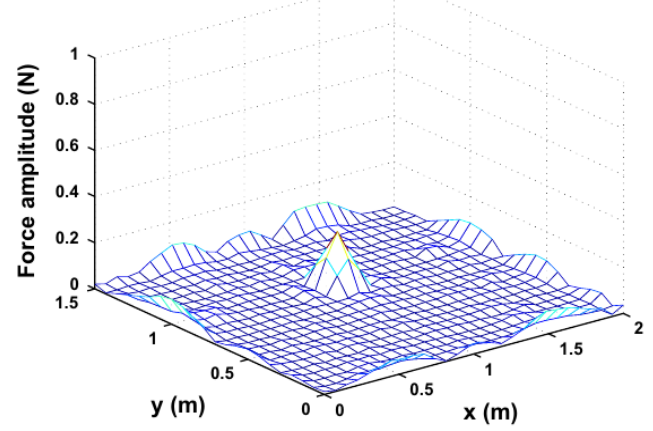

(a)

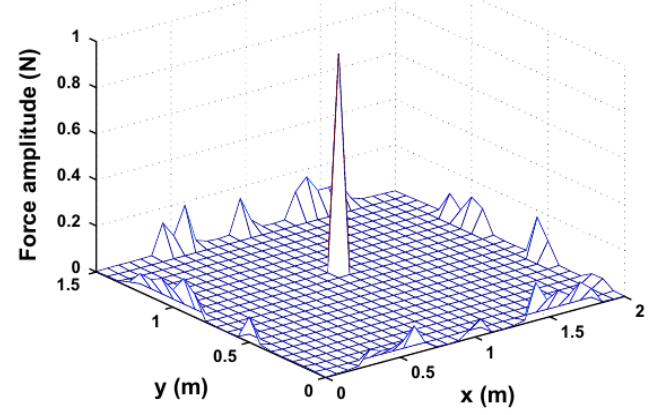

(b)

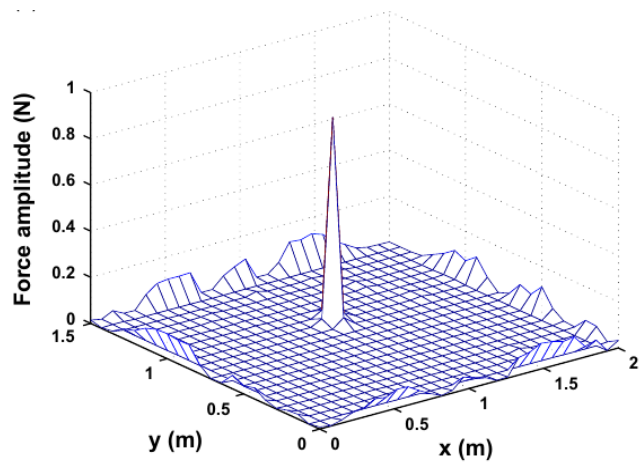

(c)

Figure 5: Identification of the force vector $\widehat{\mathbf{F}}_{\mathbf{c}}$ at $350 \mathrm{~Hz}$ from corrupted data and with regularization - Identified force vector using (a) a Tikhonov regularization or a sparse regularization with $(\mathrm{b})(\mathrm{p}, \mathrm{q})=(2,1)$ and $(\mathrm{c})(\mathrm{p}, \mathrm{q})=(1.9,1.1)$ 


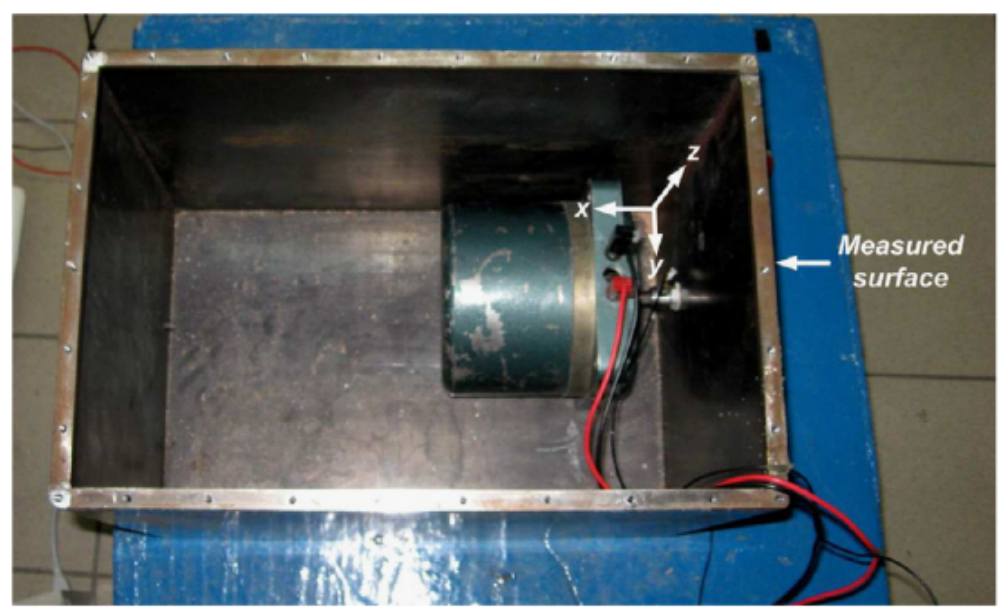

Figure 6: Experimental set-up

carried out with a scanning laser vibrometer on a grid of $19 \times 22$ points along $\mathrm{y}$ and $\mathrm{z}$ directions respectively. using the force signal as a phase reference. Hence, the distance between two measurement points is $17 \mathrm{~mm}$ in average. In all the subsequent identifications, the measured vibration velocity field has been normalized to the force signal delivered by the force sensor. In other words, the transfer functions between the vibration signals and the force signal are used as input data of the identification problem. Technically speaking, these transfer functions correspond to the transfer function estimates $H_{1}$ derived from standard signal processing techniques. Furthermore, to be consistent with the formulation presented in section 2, each transfer function has been numerically integrated by the division of its values by $j \omega$ so as to estimate the transfer functions between the displacement signals and the force signal. It is important to note that the experimental procedure described above is established for validation purposes only insofar as, by doing so, the amplitude of the identified point force F0 should be equal to 1. Fi- 
nally, it should be noted that choosing the force signal as a phase reference places us in a favorable experimental configuration. Indeed, in an industrial context, a vibration sensor located in the studied area would be used as a phase reference since measuring directly the force injected in the structure is generally impossible.

Regarding now the definition of the FE mesh used to model the dynamic behavior of the excited surface, it has been designed so as to perfectly match the measurement mesh. Hence, it is made up of 378 shell elements. The corresponding FE model with free boundary conditions has then been used to compute the transfer functions matrix $\mathbf{H}$, considering that transverse motions are the only available data. In this experimental validation, the structural damping is a priori unknown, even if it could be assessed from the vibration measurements. However, instead of neglecting the structural damping, its value is set to 0.1 percent to avoid numerical difficulties when calculating $\mathbf{H}$ from Eq. (4).

Fig. 7(a) presents the force vector identified at $350 \mathrm{~Hz}$ when no regularization is applied. As expected, the source identification fails. Here again, the first idea to stabilize the identification problem is to use the standard Tikhonov regularization [see Fig. 7(b)]. As previously observed, the singularity related to the point force is over-smoothed, while the distribution of the identified reaction forces seems consistent with what is expected when analyzing the experimental set-up. To improve this result, prior information on the measurement noise and the spatial distribution of sources has to be used. 
Assuming that the measurement noise is similar to that modeled in section 4 and the force vector to identify is sparse, one obtains after 74 iterations the results presented in Fig. 7(c) corresponding to a generalized Tikhonov regularization with $(p, q)=(1.9,0.5)$ and $\mathbf{L}=\mathbf{I}$. As expected, the point force is well identified, since $\widehat{F}_{0}=0.975$ and $\left(\widehat{\mathrm{x}}_{0}, \widehat{\mathrm{y}}_{0}, \widehat{\mathrm{z}}_{0}\right)=(0 \mathrm{~m}, 0.1026 \mathrm{~m}, 0.0875 \mathrm{~m})$. However, spurious point forces appear at boundaries, indicating that our a priori on the spatial distribution of sources is not suited to identify the boundary conditions. Finally, the best compromise between the smoothness of reacting forces at boundaries and the singularity of the point force is achieved after 65 iterations by setting $(p, q)=(1.9,1.1)$ and $\mathbf{L}=\mathbf{I}$ [see Fig. $7(\mathrm{~d})$ ]. In this situation, the spatial distribution of reacting forces is in accordance with that obtained with the standard Tikhonov regularization, whereas the amplitude of the point force is spread around its actual location. Incidentally, the amplitude of the identified point force is under-estimated. Nevertheless, the actual amplitude of the point force can be retrieved by performing a spatial summation of the force distribution around the identified peak location. Such a summation compensates for the dispersion of the point force amplitude. In the present case, the spatial summation is performed by taking all the nodes included in the area defined in Fig. 8. By doing so, the estimated point force amplitude is $\widehat{\mathrm{F}}_{0}=1.08$, which approaches the target value of 1 .

Furthermore, as for the numerical validation, it should be noted that the L-curve used to automatically select the trade- off parameter has the typical aspect of an L-curve as presented in Fig. 9. 


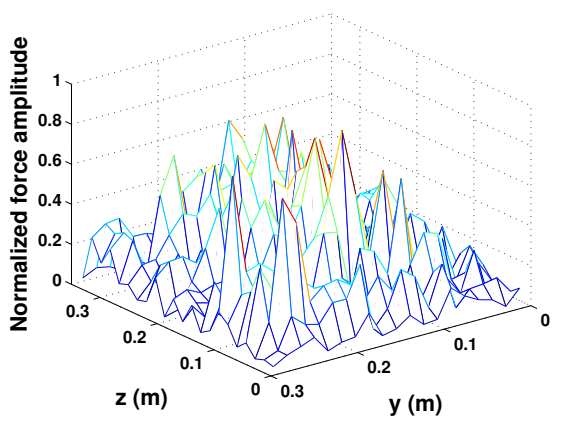

(a)

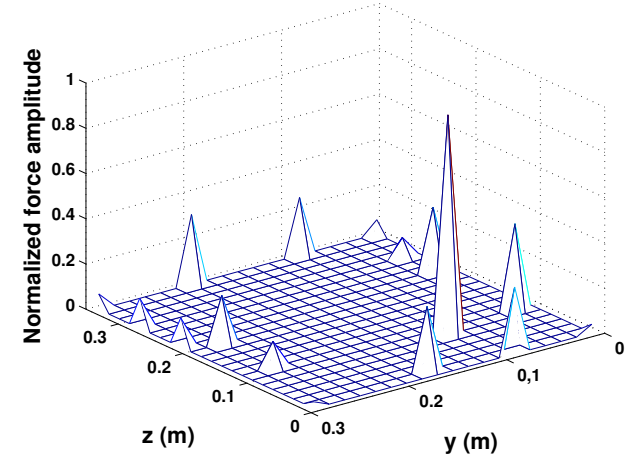

(c)

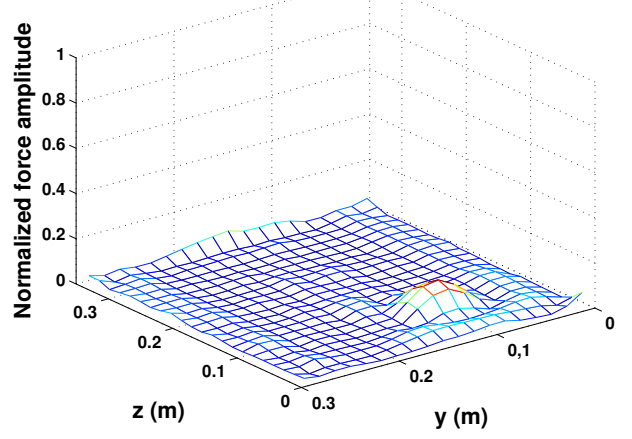

(b)

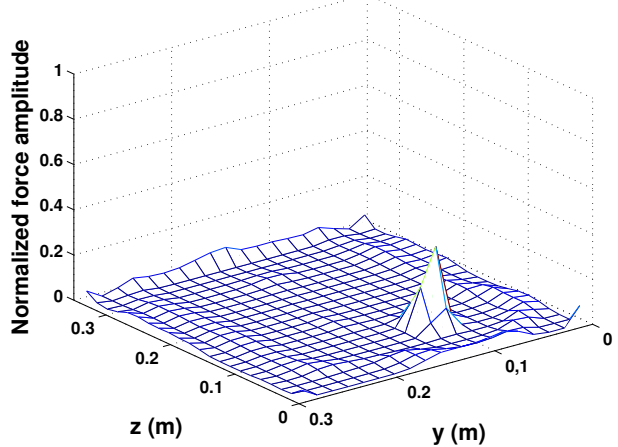

(d)

Figure 7: Experimental identification of the force vector $\widehat{\mathbf{F}}_{\mathbf{c}}$ at $350 \mathrm{~Hz}$ using (a) a Tikhonov regularization or a sparse regularization with $(\mathrm{b})(\mathrm{p}, \mathrm{q})=(1.9,0.5)$ and $(\mathrm{c})(\mathrm{p}, \mathrm{q})=(1.9,1.1)$ 


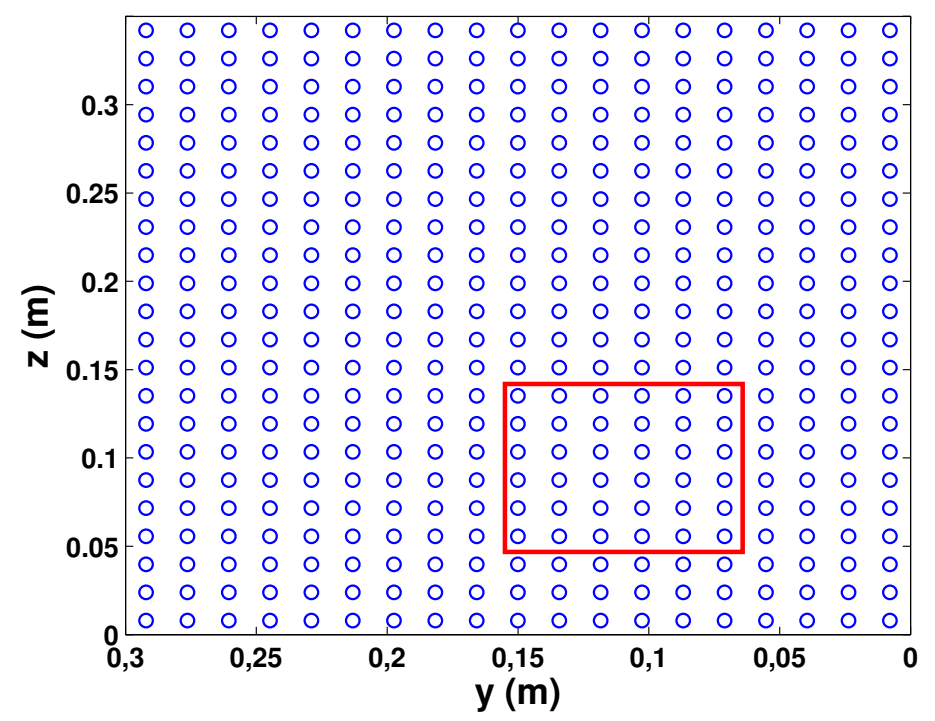

(a)

Figure 8: Definition of the summation area. $(\bigcirc)$ identification nodes and $(-)$ contour of the summation area 


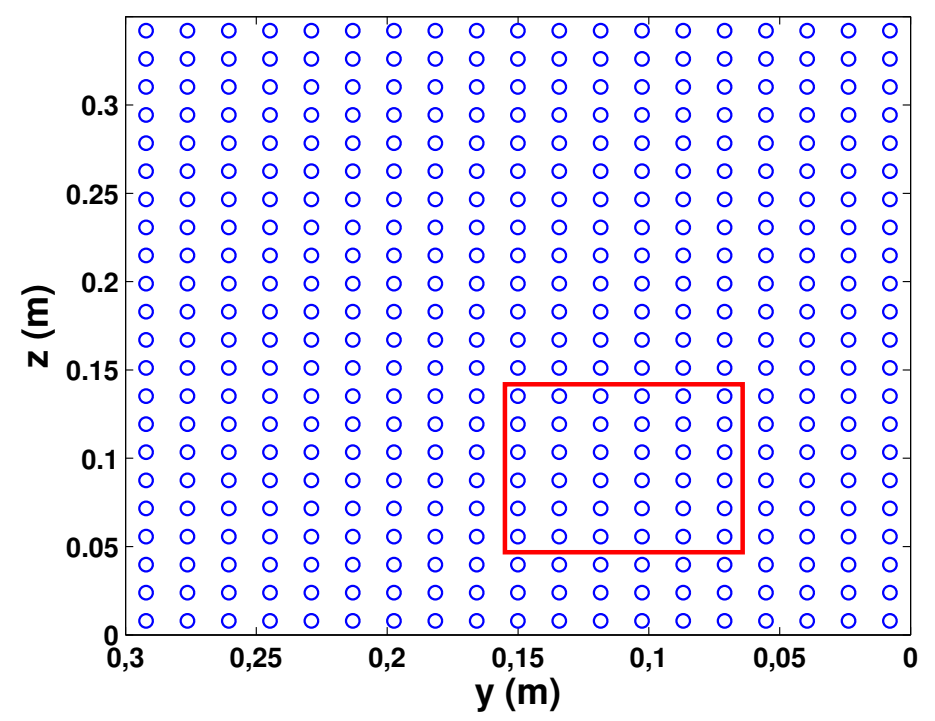

(a)

Figure 9: Shape of the L-curve obtained for the experimental test case

At this stage, it is interesting to provide the results obtained at other frequencies given in Table 2 when using the proposed generalized Tikhonov regularization for $(p, q)=(1.9,1.1)$ to verify whether the analysis performed previously remains valid. Fig. 10 presents the force vector identified at 225 $\mathrm{Hz}, 650 \mathrm{~Hz}, 1350 \mathrm{~Hz}$ and $1650 \mathrm{~Hz}$. For each case, it can be pointed out that the reaction forces are consistent with our expectation and the location of the point force is properly estimated, while its amplitude is distributed around the actual location. Consequently, these results are in agreement with those obtained at $350 \mathrm{~Hz}$.

Finally, for the sake of completeness, the amplitude of the identified point force is calculated for each frequency studied previously by performing a spatial summation in the area defined in Fig. 8. The corresponding results are 


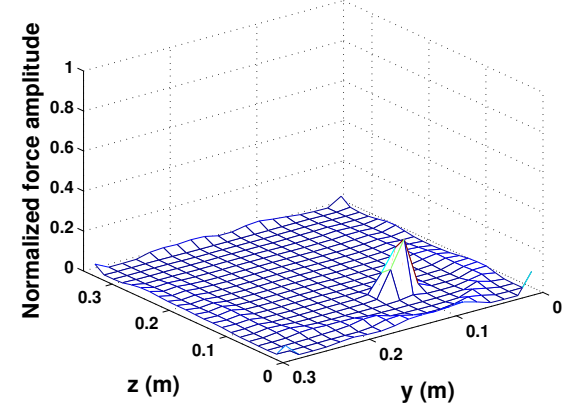

(a)

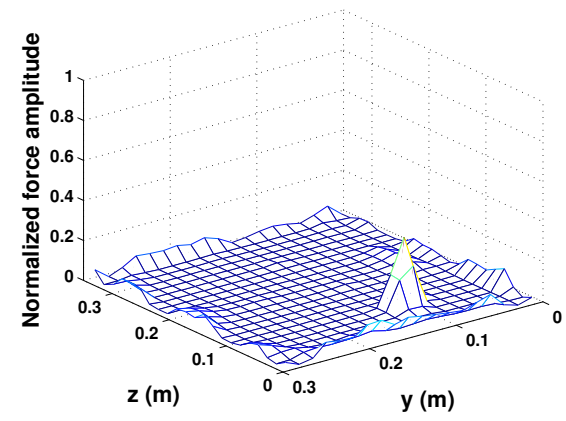

(c)

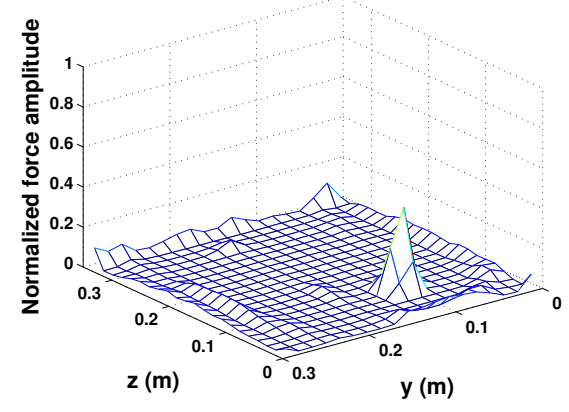

(b)

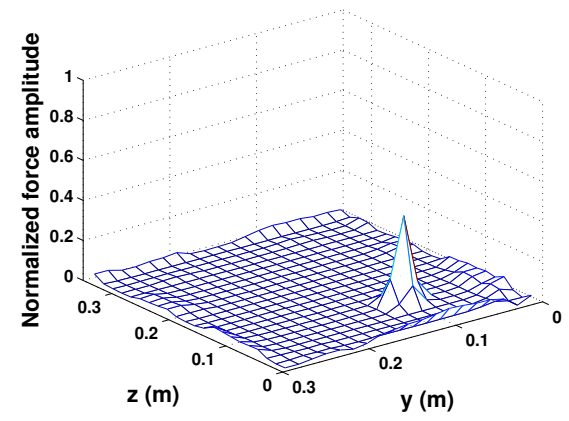

(d)

Figure 10: Experimental identification of the force vector $\widehat{\mathbf{F}_{\mathbf{c}}}$ using a generalized Tikhonov regularization with $(p, q)=(1.9,1.1)$ at (a) $225 \mathrm{~Hz}$, (b) $650 \mathrm{~Hz}$, (c) $1350 \mathrm{~Hz}$ and (d) 1650 $\mathrm{Hz}$ 
summarized in Table 3. In particular, it should be noted that the estimated point force amplitude is relatively close to the target value of 1 for each studied frequency, with a maximal error of $12 \%$ observed at $650 \mathrm{~Hz}$. This indicates that the proposed identification procedure allows obtaining solutions consistent with the distribution and the amplitudes of actual sources.

Table 3: Summary of the experimental results for $(p, q)=(1.9,1.1)$ at various frequencies

\begin{tabular}{lll}
\hline Frequency $(\mathrm{Hz})$ & $\widehat{\boldsymbol{F}}_{\mathbf{0}}$ & Figure ID \\
\hline 225 & 0.95 & Fig. 10(a) \\
350 & 1.08 & Fig. $7(\mathrm{~d})$ \\
650 & 1.12 & Fig. 10(b) \\
1350 & 1.04 & Fig. 10(c) \\
1650 & 1.11 & Fig. 10(d) \\
\hline
\end{tabular}

The presented experimental results therefore tend to confirm that the quality of the source identification is closely related to prior information, reflecting the expectation of the experimenter according to his knowledge of the whole system (measurement devices and structure under test). In other words, if no prior knowledge is assumed, the probability of good identification is weak.

\section{Conclusion}

In the present study, the problem of source identification using a generalized Tikhonov regularization was investigated. This approach allows introducing prior information on the measurement noise corrupting the data as well as on the nature of sources to identify, making possible to exploit the a 
priori knowledge of the experimenter on the system to identify. From a mathematical point of view, such a formulation can lead to solve either convex or non-convex problems. To deal with both problems properly, a Generalized Iteratively Reweighted Least-Squares algorithm has been proposed. It requires the knowledge of the vibration field measured over the real structure and the transfer functions matrix that characterizes the dynamic behavior of the structure under test. Several strategies can be developed to obtain the transfer functions matrix. In this study, it has been calculated from a FE model of the structure by taking into account only the measurable dofs. From these input data, both subsequent numerical and experimental validations have pointed out the crucial role of prior information in the source identification process as well as on the performance of the GIRLS algorithm. In particular, it can be emphasized that such a regularization process gives the most probable solution given prior information on the measurement noise and the spatial distribution of sources to identify. Consequently, if prior information properly reflects the actual measurement noise (additive Gaussian, impulsive, multiplicative, etc.), as well as the nature of the actual sources to identify (distributed and/or localized), then the convergence of the GIRLS algorithm is obtained within a few iterations.

[1] K. Stevens. Force identification problem - an overview. In Proceedings of the SEM Spring Meeting, Houston, USA, 1987.

[2] B. J. Dobson and E. Rider. A review of the indirect calculation of excitation forces from measured structural response data. Journal of Mechanical Engineering Science, 204:69-75, 1990. 
[3] M. Blau. Inverse force synthesis: State of the art and future research. In Proceedings of Inter-Noise 2000, Nice, France, 2000.

[4] S. Vanlanduit, P. Guillaume, B. Cauberghe, E. Parloo, G. De Sitter, and P. Verboven. On-line identification of operational loads using exogenous inputs. Journal of Sound and Vibration, 285 (1-2):267-379, 2005.

[5] T. Otsuka, T. Okada, T. Ikeno, K. Shiomi, and M. Okuma. Force identification of an outboard engine by experimental means of linear structural modeling and equivalent force transformation. Journal of Sound and Vibration, 308 (3-5):541-547, 2007.

[6] Q. Leclere, C. Pezerat, B. Laulagnet, and L. Polac. Indirect measurement of main bearing loads in an operating diesel engine. Journal of Sound and Vibration, 286 (1-2):341-361, 2005.

[7] M. H. A. Janssens and J. W. Verheij. A pseudo-forces methodology to be used in characterization of structure-borne sound sources. Applied Acoustics, 61:285-308, 2000.

[8] P. Guillaume, E. Parloo, P. Verboven, and G. De Sitter. An inverse method for the identification of localized excitation sources. In Proceedings of the 20th International Modal Analysis Conference, Los Angeles, USA, 2002.

[9] E. Parloo, P. Verbiven, P. Guillaume, and M. Van Overmeire. Force identification by means of in-operation modal models. Journal of Sound and Vibration, 262 (1):161-173, 2003. 
[10] C. Pezerat and J. L. Guyader. Two inverse methods for localization of external sources exciting a beam. Acta Acustica, 3:1-10, 1995.

[11] S.R. Ibrahim, A. Fregolent, and A. Sestieri. Structural force identification at unmeasured locations. In Proceedings of the 14th International Modal Analysis Conference, Dearborn, USA, 1996.

[12] Y. Zhang and J. A. Mann III. Examples of using structural intensity and the force distribution to study vibrating plates. Journal of the Acoustical Society of America, 99 (1):354-361, 1996.

[13] C. Renzi. Identification Expérimentale de Sources vibratoires par Résolution du probleme Inverse modelisé par un opérateur Eléments Finis local (Vibratory sources identification by solving the inverse problem modeled by a local Finite Element operator). PhD Thesis, INSA de Lyon, 2011.

[14] C. Renzi, C. Pezerat, and J.-L. Guyader. Vibratory source identification by using the finite element model of a subdomain of a flexural beam. Journal of Sound and Vibration, 332:545-562, 2013.

[15] Q. Leclere and C. Pezerat. Vibration source identification using corrected finite difference schemes. Journal of Sound and Vibration, 331:1366-1377, 2012.

[16] R. E. Powell and W. Seering. Multichannel structural inverse filtering. Journal of Vibration, Acoustics, Stress and Reliability in Design, 106:2228,1984 .

[17] J. W. Verheij. Inverse and reprocity methods for machinery noise source 
characterization and sound path quantification part 1 : Sources. International Journal of Acoustics and Vibration, 2 (1):11-20, 1997.

[18] M. H. A. Janssens, J. W. Verheij, and D. J. Thompson. The use of an equivalent forces method for the experimental quantification of structural sound transmission in ships. Journal of Sound and Vibration, 226 (2):305-328, 1999.

[19] A. N. Thite and D. J. Thompson. The quantification of structure-borne transmission paths by inverse methods. part 1 : Improved singular value rejection methods. Journal of Sound and Vibration, 264 (2):411-431, 2003.

[20] Y. Liu and W. Steve Shepard Jr. Reducing the impact of measurement errors when reconstructing dynamic forces. Journal of Vibration and Acoustics, 128:586-593, 2006.

[21] H. R. Busby and D. M. Trujillo. Optimal regularization of an inverse dynamics problem. Computers \&3 Structures, 63 (2):243-248, 1997.

[22] A. N. Thite and D. J. Thompson. The quantification of structure-borne transmission paths by inverse methods. Part 2: Use of regularization techniques. Journal of Sound and Vibration, 264 (2):433-451, 2003.

[23] J. Flemming. Generalized Tikhonov regularization: Basic theory and comprehensive results on convergence rates. $\mathrm{PhD}$ thesis, Technische Universität Chemnitz, 2011. 
[24] M. Green. Statistics of Images, the TV Algorithm of Rudin-OsherFatemi for Image Denoising and an Improved Denoising Algorithm. Technical report, UCLA, 2002.

[25] T. Le, R. Chartrand, and T. J. Asaki. A variational approach to reconstructing images corrupted by poisson noise. Journal of Mathematical Imaging and Vision, 27:257-263, 2007.

[26] K. Lange. Sound Source Reconstruction with Covariance Matrix Fitting. Master thesis, Technical University of Denmark, 2010.

[27] S. Boyd and L. Vandenberghe. Convex optimization. Cambridge University Press, 2004.

[28] M. Nikolova. A variational approach to remove outliers and impulse noise. Journal of Mathematical Imaging and Vision, 20 (1-2):99-120, 2004 .

[29] M. Nikolova. Minimizers of cost-functions involving non-smooth datafidelity terms. application to the processing of outliers. SIAM Journal on Numerical Analysis, 40 (3):965-994, 2002.

[30] L. Rudin, S. Osher, and E. Fatemi. Nonlinear total variation based noise removal algorithms. Physica D, 60:259-268, 1992.

[31] R. Tibshirani. Regression shrinkage and selection via the lasso. Journal of the Royal Statistical Society, 58 (1):267-288, 1996.

[32] A. Chambolle. Total variation minimization and a class of binary mrf models. Lecture Notes in Computer Science, 3757/2005:136-152, 2005. 
[33] H. Fu, K. Ng, M. Nikolova, and J. L . Barlow. Efficient minimization methods of mixed $l_{2}-l_{1}$ and $l_{1}-l_{1}$ norms for image restoration. SIAM Journal on Scientific Computing, 27 (6):1881-1902, 2006.

[34] J. Bioucas-Dias, M. Figueiredo, and J. Oliveira. Total variation image deconvolution: A majorization-minimization approach. In Proccedings of International Conference on Acoustics, Speech ans Signal Processing, Toulouse, France, 2006.

[35] R. Chartrand and V. Stavena. Nonconvex regularization for image segmentation. In Proccedings of International Conference on Image Processing, Computer Vision and Pattern Recognition (IPCV) 200\%, Las Vegas, USA, 2007.

[36] M. Nikolova, M. K. Ng, S. Zhang, and W.-K. Ching. Efficient reconstruction of piecewise constant images using nonsmooth nonconvex minimization. SIAM Journal on Imaging Sciences, 1 (1):2-25, 2008.

[37] M. Hintermuller and T. Wu. Nonconvex TV ${ }^{\mathrm{q}}$-models in image restoration: Analysis and a trust-region regularization based superlineraly convergent solver. Technical report, Institute of Mathematics ans Scientific Computing, University of Graz, 2011.

[38] P. Rodriguez and B. Wohlberg. An iteratively weighted norm algorithm for total variation regularization. In Proceedings of the 2006 Asilomar Conference on Signals, Systems, and Computers, Pacific Grove, USA, 2006. 
[39] N. Bissantz, L. Dumbgen, L. Munk, and B. Stratmann. Convergence analysis of generalized iteratively reweighted least squares algorithms on convex function spaces. SIAM Journal on Optimization, 19:1828$1845,2009$.

[40] M.-J. Lai and J. Wang. An unconstrained $l_{q}$ minimization with $0<q \leq 1$ for sparse solution of under-determined linear systems. SIAM Journal on Optimization, 21 (1):82, 2011.

[41] C. L. Lawson. Contributions to the Theory of Linear Least Maximum Approximation. PhD Thesis, University of California, 1961.

[42] P. J . Green. Iteratively reweighted least squares for maximum likelihood estimation, and some robust and resistant alternatives. Journal of the Royal Statistical Society. Series B (Methodological), 46 (2):149-192, 1984.

[43] R. Chartrand and W. Yin. Iteratively reweighted algorithms for compressive sensing. In Proccedings of International Conference on Acoustics, Speech ans Signal Processing, Toulouse, France, 2008.

[44] G. Chardon and L. Daudet. Narrowband source localization in an unknown reverberant environment using wavefield sparse decomposition. In Proccedings of International Conference on Acoustics, Speech and Signal Processing 2012, Kyoto, Japan, 2012.

[45] C. Renzi, C. Pezerat, and J.-L. Guyader. Identification of vibration excitation using a regularized finite element operator and a deconvolution post-process. In Proceedings of Acoustics 2012, Nantes, France, 2012. 
[46] E. Balmes. Sensors, degrees of freedom and generalized modeshape expansion methods. In Proceedings of International Modal Analysis Conference, Kissimee, USA, 1999.

[47] C. Renzi, C. Pezerat, and J.-L. Guyader. Identification des sources vibratoires en utilisant un opérateur eléments finis (Source identification using a finite element operator). In Proceedings of $10^{\text {ème }}$ Congrès Français d'Acoustique, Lyon, France, 2010.

[48] C. Renzi, C. C. Pezerat, and J.-L. Guyader. Vibration sources identification using vibratory measurements injected in a local finite element model. In Proceedingd of ISMA 2010, 2010.

[49] A. Batou and C. Soize. Experimental identification of stochastic processes using an uncertain computational non-linear dynamical model. Journal of Physics: Conference Series, 135:012014, 2008.

[50] M. Corus. Force identification using a statically completed reduced model deriving from tests. Journal of Sound and Vibration, 329:41494165, 2010.

[51] S. E. Dosso and M. J. Wilmut. Bayesian focalization: Quantifying source localization with environmental encertainty. Journal of the Acoustical Society of America, 121 (5):2567-2574, 2007.

[52] J. Antoni. A Bayesian approach to sound source reconstruction: Optimal basis, regularization, and focusing. Journal of the Acoustical Society of America, 131 (4):2873-2890, 2012. 
[53] M. M. Lee. Bayesian reconstruction in emission tomography using Gibbs Priors. PhD Thesis, Yale University, 1994.

[54] P. Rodriguez and B. Wohlberg. Efficient minimization method for a generalized total variation functional. IEEE Transactions on Image Processing, 18 (2):322-332, 2009.

[55] J. A. Scales and A. Gersztenkorn. Robust methods in inverse theory. Inverse Problems, 4:1071-1091, 1988.

[56] M. Grasmair. Non-convex sparse regularization. Journal of Mathematical Analysis and Applications, 365 (1):19-28, 2010.

[57] O. Scherzer. The use of Morozov's discrepancy principle for Tikhonov regularization for solving nonlinear ill-posed problems. Computing, 51:45-60, 1993.

[58] G. H. Golub, M. Heath, and G. Wahba. Generalized cross-validation as a method for choosing a good ridge parameter. Technometrics, 21 (2):215-223, 1979.

[59] Y. Lin and B. Wohlberg. Application of the upre method to optimal parameter selection for large scale regularization problems. In IEEE Southwest Symposium on Image Analysis and Interpretation, Santa FE, USA, 2008.

[60] P. C. Hansen. Rank-Deficient and Discrete Ill-Posed Problems: Numerical Aspects of Linear Inversion. SIAM, 1998. 
[61] M. Aucejo, N. Totaro, and J.-L. Guyader. Identification of source velocities on 3D structures in non-anechoic environments: Theoretical background and experimental validation of the inverse patch transfer functions method. Journal of Sound and Vibration, 329 (18):3691-3708, 2010.

[62] Q. Leclere. Acoustic imaging using under-determined inverse approaches: Frequency limitations and optimal regularization. Journal of Sound and Vibration, 321:605-619, 2009. 